\title{
Classification of effective operators for interactions between the Standard Model and dark matter
}

\section{Duch, ${ }^{a}$ B. Grzadkowski ${ }^{a}$ and J. Wudka ${ }^{b}$}

\author{
${ }^{a}$ Faculty of Physics, University of Warsaw, \\ Hoża 69, 00-681 Warsaw, Poland \\ ${ }^{b}$ Department of Physics and Astronomy, UC Riverside, \\ Riverside, CA 92521, U.S.A. \\ E-mail: mateusz.duch@fuw.edu.pl, bohdan.grzadkowski@fuw.edu.pl, \\ jose.wudka@ucr.edu
}

ABSTRACT: We construct a basis for effective operators responsible for interactions between the Standard Model and a dark sector composed of particles with spin $\leq 1$. Redundant operators are eliminated using dim- 4 equations of motion. We consider simple scenarios where the dark matter components are stabilized against decay by $\mathbb{Z}_{2}$ symmetries. We determine operators which are loop-generated within an underlying theory and those that are potentially tree-level generated.

Keywords: Beyond Standard Model, Cosmology of Theories beyond the SM, Effective field theories

ARXIV EPRINT: 1412.0520 


\section{Contents}

1 Introduction 1

$\begin{array}{lll}2 & \nu \mathrm{SM} \text { operators } & 2\end{array}$

3 Dark operators 3

4 The effective Lagrangian $\quad 5$

4.1 The Stuckelberg mechanism 8

4.2 The Higgs mechanism 8

$4.3 \nu \mathrm{SM} \times \mathrm{DM}$ operators $\quad 9$

$\begin{array}{lll}5 & \text { Summary } & 12\end{array}$

$\begin{array}{ll}\text { A Conventions and definitions } & 12\end{array}$

B Mass generation for Abelian vector bosons $\quad 13$

$\begin{array}{ll}\text { B.1 The Stuckelberg mechanism } & 13\end{array}$

$\begin{array}{ll}\text { B.2 The Higgs mechanism } & 13\end{array}$

\section{Introduction}

Understanding the nature of dark matter (DM) is one of the most pressing current issues in astroparticle physics. Of the many hypotheses proposed, one of the most fruitful and promising is based on the assumption that DM is composed of one or more new elementary particles. This possibility has been extensively studied in a variety of specific models, most prominently in realistic supersymmetric models that are characterized by predicting that $\mathrm{DM}$ is the lightest supersymmetric particle whose mass should be in the $O(100 \mathrm{GeV})$ range.

In this paper we will be interested in constructing a model-independent description of the interactions between the dark and SM sectors using an effective Lagrangian approach. We will assume the standard sector with one doublet scalar field, but augmented by a number of right-handed neutrinos $\nu_{R}$ to allow for the possibility of Dirac neutrino masses. We denote this enlarged model as the $\nu \mathrm{SM}$.

Concerning the dark sector we allow the dark matter to be multi-component, containing fermions, scalars and massive Abelian vectors. Dark matter particles are protected against decay by a symmetry we denote $\mathcal{G}_{\text {dark }}$, and which we need not to specify at this point, but which can include discrete or continuous (local or global) subgroups; it also contains the local symmetry related to mass generation of the dark vector. It is important to note that even though the dark sector may contain several stable particles, not all have to contribute significantly to the DM relic density inferred from the WMAP and PLANCK data $[1,2]$. 
Within the scenarios we consider, the dark and $\nu \mathrm{SM}$ sectors interact through the exchange of heavy particles whose mass is much larger than the typical momentum transfer in all processes being considered and their effects decouple at low-energies. In addition, we will assume that the underlying theory is weakly coupled and renormalizable. Under these circumstances the DM- $\nu \mathrm{SM}$ interactions can be described by a series of effective operators

$$
\mathcal{O}_{\mathrm{DM}-\nu \mathrm{SM}}=\mathcal{O}_{\mathrm{DM}} \mathcal{O}_{\nu \mathrm{SM}}
$$

where $\mathcal{O}_{\mathrm{DM}}$ and $\mathcal{O}_{\nu \mathrm{SM}}$ are composed of fields belonging to the respective sectors, and it is assumed that they are invariant under corresponding symmetries. The effective Lagrangian consists of a linear combination of terms of this type. The coefficients are suppressed by appropriate powers of a heavy-physics scale $\Lambda$ (the power is determined by the dimension of $\mathcal{O}_{\mathrm{DM}-\nu \mathrm{SM}}$ ), and contain unknown dimensionless couplings that parameterize at low energies all interactions between the standard and dark sectors. In addition to the hierarchy generated by operator dimensionality it is also useful to note that some of the operators are necessarily generated by heavy-particle loops, so that their coefficients are correspondingly suppressed, the remaining operators can be generated at tree level, but whether this is the case depends on the details of the underlying theory. In this paper we will construct all operators $\mathcal{O}_{\mathrm{DM}-\nu \mathrm{SM}}$ of dimension $\leq 6$ and determine whether they can be generated at the tree level.

In constructing the effective Lagrangian, we will eliminate operators that vanish when dim-4 equations of motion are imposed, since they give no contribution to on-shell matrix elements, both in perturbation theory (to all orders) and beyond [3-8]; we call such operators redundant. A given type of heavy physics may generate a basis of operators different from the one listed below; such a basis may be transformed in the one we use by applying equations of motion.

Dim-6 effective operators for interactions between the Standard Model and DM have been already present in various contexts in the literature [9-28]. However the goal of this paper is to construct a basis of operators [29] that then could be consistently adopted to describe different aspects of DM physics.

The paper is organized as follows. In section 2 we define the model and list $\nu \mathrm{SM}$ operators up to dimension 4 . In section 3 we present dark operators needed to construct dim- 6 effective operators. Section 4 contains our main results, i.e. the basis of operators up to dimension 6. In section 5 we summarize of our findings. Appendix A specifies our conventions, while appendix B reviews mechanisms for dark vector boson mass generation.

\section{$2 \quad \nu \mathrm{SM}$ operators}

As mentioned above we will consider the Standard Model of electroweak interactions supplemented by a number of right-handed neutrinos $\nu_{R}(\nu \mathrm{SM})$; this model contains the matter fields collected in table 1 . We assume 3 quark families, 3 lepton $\mathrm{SU}(2)$ doublets and charged right-handed lepton singlets, and $n_{\nu}$ right-handed neutrinos. We use these fields to construct the gauge-invariant operators $\mathcal{O}_{\nu \mathrm{SM}}$ appearing in (1.1), which we classify according to their canonical dimension (up to $\operatorname{dim} \leq 4$ ) and number of Lorentz indices; these operators are collected in table 2 (Hermitian conjugation of operators containing fermions are 


\begin{tabular}{|c|c|c|c|c|c|c|c|}
\hline & \multicolumn{6}{|c|}{ fermions } & scalars \\
\hline field & $l_{L p}^{j}$ & $e_{R p}$ & $\nu_{R k}$ & $q_{L p}^{\alpha j}$ & $u_{R p}^{\alpha}$ & $d_{R p}^{\alpha}$ & $\varphi^{j}$ \\
\hline hypercharge $Y$ & $-\frac{1}{2}$ & -1 & 0 & $\frac{1}{6}$ & $\frac{2}{3}$ & $-\frac{1}{3}$ & $\frac{1}{2}$ \\
\hline
\end{tabular}

Table 1. $\nu \mathrm{SM}$ matter field content and their hypercharge quantum numbers. Weak isospin, colour and generation indices are denoted by $j=1,2, \alpha=1,2,3$ and $p=1,2,3$ respectively. We assume the presence of $n_{\nu}$ right-handed neutrinos, so $k=1, \ldots, n_{\nu}$.

\begin{tabular}{|c|c|c|c|}
\hline $\operatorname{dim}$ & scalars & vectors & tensors \\
\hline $3 / 2$ & $\nu_{R}$ & - & - \\
\hline 2 & $\varphi^{\dagger} \varphi$ & - & $\left(\stackrel{\sim}{B}_{\mu \nu}\right.$ \\
\hline $5 / 2$ & $\bar{l} \tilde{\varphi}$ & $\partial_{\mu} \nu_{R}$ & - \\
\hline 3 & $\nu_{R}^{T} C \nu_{R}$ & $\begin{array}{l}\bar{\psi} \gamma_{\mu} \psi, i \varphi^{\dagger} \overleftrightarrow{D}_{\mu} \varphi \\
\partial_{\mu}\left(\varphi^{\dagger} \varphi\right), \partial^{\mu} B_{\mu \nu}\end{array}$ & $\nu_{R}^{T} C \sigma_{\mu \nu} \nu_{R}, \partial_{\rho} \stackrel{(\sim)}{B}_{\mu \nu}$ \\
\hline $7 / 2$ & $\varphi^{\dagger} \varphi \nu_{R}, \partial^{2} \nu_{R}$ & $\bar{l} D_{\mu} \tilde{\varphi},\left(D_{\mu} \bar{l}\right) \tilde{\varphi}$ & $\partial_{\mu} \partial_{\nu} \nu_{R}, \nu_{R} \stackrel{(\sim)}{B}_{\mu \nu}$ \\
\hline 4 & $\begin{array}{l}\left(D_{\mu} \varphi\right)^{\dagger} D^{\mu} \varphi, \varphi^{4}, \bar{\psi} \not D \psi \\
\bar{l} \nu_{R} \tilde{\varphi}, \bar{l} e \varphi, \bar{q} u \tilde{\varphi}, \bar{q} d \varphi \\
(\sim) \\
X_{\mu \nu} X^{\mu \nu},\left(D_{\mu} \bar{\psi}\right) \gamma^{\mu} \psi \\
\varphi^{\dagger} D_{\mu} D^{\mu} \varphi,\left(D_{\mu} D^{\mu} \varphi\right)^{\dagger} \varphi\end{array}$ & $\begin{array}{l}\nu_{R}^{T} C \partial_{\mu} \nu_{R}, \nu_{R}^{T} C \gamma_{\mu} \not \partial \nu_{R} \\
\nu_{R}^{T} C \gamma^{\mu} l \varepsilon \varphi\end{array}$ & $\begin{array}{l}\stackrel{(\sim)}{X}_{\mu \rho} X_{\nu}{ }^{\rho}, \partial^{2} \stackrel{(\sim)}{B}_{\mu \nu}, \varphi^{\dagger} \stackrel{(\sim)}{W}_{\mu \nu} \varphi, \varphi^{\dagger} \stackrel{(\sim)}{B}_{\mu \nu} \varphi \\
\partial_{\mu} \partial_{\nu}\left(\varphi^{\dagger} \varphi\right), \partial_{\mu}\left(i \varphi^{\dagger} \stackrel{D}{D}_{\nu} \varphi\right),\left(D_{\mu} \varphi\right)^{\dagger} D_{\nu} \varphi \\
\bar{\psi} D_{\mu} \gamma_{\nu} \psi, \partial_{\mu}\left(\bar{\psi} \gamma_{\nu} \psi\right), \partial_{\mu} \partial^{\rho} B_{\rho \nu} \\
\bar{l} \sigma^{\mu \nu} \nu_{R} \tilde{\varphi}, \bar{l} \sigma^{\mu \nu} e \varphi, \bar{q} \sigma^{\mu \nu} u \tilde{\varphi}, \bar{q} \sigma^{\mu \nu} d \varphi\end{array}$ \\
\hline
\end{tabular}

Table 2. $\nu \mathrm{SM}$ operators that are singlets of $\mathrm{SU}(3)_{C} \times \mathrm{SU}(2)_{L} \times \mathrm{U}(1)_{Y}$ in different Lorentz group representations. $X_{\mu \nu}$ stands for $B_{\mu \nu}, W_{\mu \nu}^{I}$ or $G_{\mu \nu}^{A}, \psi \in\left\{l, \nu_{R}, e, q, u, d\right\}$. This list includes operators that are total derivatives, equations of motion were not adopted at this stage.

not listed separately but should be included when constructing the effective Lagrangian in order to ensure it is Hermitian). It should also be noted that, in the presence of fermionic fields there exists operators where Dirac matrices might appear between the two factors in (1.1). We also use the fact that four-fermion operators can always be rearranged into the form (1.1) by using Fierz transformations. All $\nu$ SM fields are assumed to be singlets under symmetries stabilizing dark fields.

At this stage, we retain terms that are total derivatives and also we do not apply equations of motion at this point (that will be done when constructing the effective Lagrangian).

\section{Dark operators}

In this section we construct the list of operators ${ }^{1}$ up to $\operatorname{dim} 4$, that consist of DM fields: a real scalar $\Phi$, left and right chiral fermions $\Psi_{L}, \Psi_{R}$ and an Abelian vector field $V_{\mu}$. For

\footnotetext{
${ }^{1}$ We omit Lorentz vectors and symmetric tensors of dim- 4 , because the $\nu \mathrm{SM}$ does not contain corresponding operators of $\operatorname{dim} 2$, so they would be irrelevant while looking for effective operators $\nu \mathrm{SM} \times \mathrm{DM}$ up to $\operatorname{dim} 6$.
} 


\begin{tabular}{|c|l|l|l|}
\hline $\operatorname{dim}$ & scalars & vectors & tensors \\
\hline 2 & $\Phi^{2}$ & - & - \\
\hline $5 / 2$ & $\Phi N_{R}$ & - & - \\
\hline 3 & $\Psi^{T} C \Psi$ & $\Phi \partial_{\mu} \Phi, \bar{\Psi} \gamma_{\mu} \Psi, \phi^{*} D_{\mu} \phi+$ H.c. & $\Psi^{T} C \sigma_{\mu \nu} \Psi$ \\
\hline $7 / 2$ & - & $\Phi \partial_{\mu} N_{R}, N_{R} \partial_{\mu} \Phi$ & - \\
\hline 4 & $\bar{\Psi} \not \partial \Psi,\left(\partial_{\mu} \bar{\Psi}\right) \gamma^{\mu} \Psi$, & do not contribute to $\mathcal{O}_{\nu \mathrm{SM}}$ & $\partial_{\mu}\left(\bar{\Psi} \gamma_{\nu} \Psi\right), \bar{\Psi} \partial_{\mu} \gamma_{\nu} \Psi$, \\
& $\Phi^{4}, \partial_{\mu} \Phi \partial^{\mu} \Phi, \Phi \partial^{2} \Phi$, & of dim $\leq 6$. & $\left(D_{\mu} \phi\right)^{*} D_{\nu} \phi+$ H.c. \\
& $\left(\sim^{2}{ }_{\mu \nu} V^{\mu \nu}, \mathcal{V}_{\mu} \mathcal{V}^{\mu}\right.$, & \\
& $\left(D_{\mu} \phi\right)^{*} D^{\mu} \phi, \phi^{*} D_{\mu} D^{\mu} \phi+$ H.c. & & \\
\hline
\end{tabular}

Table 3. DM operators built of $\Phi, \Psi \in\left\{\Psi_{L}, \Psi_{R}, N_{R}\right\}$ and $V_{\mu}$ symmetric under (3.1); note the presence of operators containing $N_{R}$ and $\Phi$, allowed by the assumption of their both being odd under $\left(\mathbb{Z}_{2}\right)_{\Phi}$. For the sake of dark-sector gauge invariance the table contains also the vector field $\mathcal{V}_{\mu}$ defined in (4.12) (highlighted in red) and covariant derivatives of the scalar field $\phi$ (blue). They are relevant when the vector boson mass is generated through the Stuckelberg and Higgs mechanisms, respectively. Vector operators and symmetric tensors of dim- 4 are not listed, because we are interested in the $\nu \mathrm{SM} \times \mathrm{DM}$ operators up to dim- 6 and the $\nu \mathrm{SM}$ does not contain vector operators nor symmetric tensors with dimension less or equal 2.

simplicity, we assume that the symmetry stabilizing the dark fields is of the form

$$
\mathcal{G}_{\text {dark }}=\left(\mathbb{Z}_{2}\right)_{\Phi} \times\left(\mathbb{Z}_{2}\right)_{\Psi_{R}} \times\left(\mathbb{Z}_{2}\right)_{\Psi_{L}} \times\left(\mathbb{Z}_{2}\right)_{V}
$$

where the dark scalars $\Phi$ are odd with respect to the first factor and even with respect to the others, the $\Psi_{R(L)}$ are odd with respect to the second (third) factor and even with respects to the others, and similarly for the $V_{\mu}$. We also introduce a set of right-handed fermions $N_{R l}\left(l=1, \cdots, n_{N}\right)$ that transform in the same way as $\Phi$ under $\mathcal{G}_{\text {dark }}$. As we will show shortly, their presence allows for Yukawa interactions involving DM and $\nu_{R}$, which might be relevant for DM phenomenology. As a consequence of these assumptions the lightest particle in each of these dark sectors $\left(\Phi\right.$ and $N_{R}, \Psi_{L}, \Psi_{R}, V_{\mu}$ ) is stable separately and the effective Lagrangian will not contain terms having odd number of fields from any sector.

In appendix B we review two procedures for generating the mass of Abelian vector bosons $V_{\mu}$ : the Stuckelberg and Higgs mechanisms. The dark sector operators for both mechanisms are collected in table 3. Within the Stuckelberg approach, the U(1) gauge invariance requires that the $V_{\mu}$ appears only through the operator $\mathcal{V}_{\mu}$ or the field strength $V_{\mu \nu}$; for the Higgs approach $V_{\mu}$ appears only within the covariant derivatives of the complex scalar field $\phi$ (see appendix B). It should be stressed that $\phi$ is not a dark field (as it is explained in the appendix $|\phi|$ belongs to the heavy sector), nevertheless we retain $\phi$ in the table to ensure manifest gauge invariance. Note that all operators contained in table 3 are neutral under $\mathcal{G}_{\text {dark }}$. Since all the dark fields are assumed to be singlets under $\nu$ SM gauge symmetries, so are the operators contained in the table. 


\section{The effective Lagrangian}

In the scenario being considered, the full theory contains not only the dark and standard sectors, but also a heavy sector responsible for generating effective operators, the theory is assumed to be weakly coupled, and the scale of heavy physics $\Lambda$ is assumed to be substantially larger than the electroweak scale $v \simeq 246 \mathrm{GeV}$. At energies significantly below $\Lambda$ the dynamical content of such a theory is well described by an effective Lagrangian obtained by "integrating out" the heavy degrees of freedom, and which takes the form

$$
\mathcal{L}=\mathcal{L}^{(4)}+\frac{1}{\Lambda} \sum_{k} C_{k}^{(5)} O_{k}^{(5)}+\frac{1}{\Lambda^{2}} \sum_{k} C_{k}^{(6)} O_{k}^{(6)}+\cdots
$$

where each term $O_{k}^{(n)}$ is of the form (1.1) and is multiplied by an unknown dimensionless (Wilson) coefficient $C_{k}^{(n)}$. It should be stressed that the right-handed neutrinos $\nu_{R}$ belong to the light sector, so the scale of their mass must be $\ll \Lambda$; in the following we will assume that it is at most of the order of $v$.

The effective Lagrangian is a useful tool for parameterizing the low-energy effects of a theory in a consistent and controlled way. In the case where the low-energy theory is described by the SM the full set of such operators up to dim- 6 was constructed in [30] and then refined and systematized in [31, 32].

As we have already mentioned, we allow in the dark sector for the presence of $N_{R}$, right-handed fermions which transform the same way as $\Phi$ under $\mathcal{G}_{\text {dark }}$. As we will shortly demonstrate, this choice has important consequences since then Yukawa-type interactions between the right-handed neutrinos $\nu_{R}$, the $N_{R}$ and $\Phi$ (the last two are odd under $\left(\mathbb{Z}_{2}\right)_{\Phi}$ ) are allowed $[33,34]$. This represents a new renormalizable portal coupling between the $\nu \mathrm{SM}$ and the dark sector.

Note that since the $N_{R}$ are odd under $\mathcal{G}_{\text {dark }}$, they cannot mix with the $\nu_{L}$ to generate a Dirac mass term; the $\nu_{R}$, however, can.

The terms of dimension $\leq 4$ in the effective Lagrangian, $\mathcal{L}^{(4)}$, consists of 3 parts

$$
\mathcal{L}^{(4)}=\mathcal{L}_{\nu \mathrm{SM}}^{(4)}+\mathcal{L}_{\mathrm{DM}}^{(4)}+\mathcal{L}_{\nu \mathrm{SM} \times \mathrm{DM}}^{(4)} .
$$

The first part is the Standard Model Lagrangian with right-handed neutrinos

$$
\begin{aligned}
\mathcal{L}_{\nu \mathrm{SM}}^{(4)}= & -\frac{1}{4} G_{\mu \nu}^{A} G^{A \mu \nu}-\frac{1}{4} W_{\mu \nu}^{I} W^{I \mu \nu}-\frac{1}{4} B_{\mu \nu} B^{\mu \nu}+\left(D_{\mu} \varphi\right)^{\dagger}\left(D^{\mu} \varphi\right)+m^{2} \varphi^{\dagger} \varphi-\frac{1}{2} \lambda\left(\varphi^{\dagger} \varphi\right)^{2} \\
& +i\left(\bar{l} \not D l+\bar{\nu}_{R} \not \partial \nu_{R}+\bar{e} \not D e+\bar{q} \not D q+\bar{u} \not D u+\bar{d} \not D d\right) \\
& -\left(\bar{l} \Gamma_{\nu} \nu_{R} \tilde{\varphi}+\bar{l} \Gamma_{e} e \varphi+\bar{q} \Gamma_{u} u \tilde{\varphi}+\bar{q} \Gamma_{d} d \varphi+\text { H.c. }\right)-\frac{1}{2}\left(\nu_{R}^{T} C m_{\nu} \nu_{R}+\text { H.c. }\right)
\end{aligned}
$$

where $\Gamma_{e, u, d}$ are $3 \times 3$ matrices, $\Gamma_{\nu}$ is a $3 \times n_{\nu}$ matrix and Majorana mass $m_{\nu}$ is $n_{\nu} \times n_{\nu}$ matrix. Note that we can always choose a field basis such that $m_{\nu}$ is diagonal. 
The second term in (4.2) contains only dark fields ${ }^{2}$

$$
\begin{aligned}
\mathcal{L}_{\mathrm{DM}}^{(4)}= & \frac{1}{2} \partial_{\mu} \Phi \partial^{\mu} \Phi-\frac{1}{2} m_{\Phi}^{2} \Phi^{2}-\frac{1}{4} \kappa \Phi^{4}-\frac{1}{4} V^{\mu \nu} V_{\mu \nu}+\frac{1}{2} m_{V}^{2} V_{\mu} V^{\mu} \\
& +i\left(\bar{\Psi}_{L} \not \partial \Psi_{L}+\bar{\Psi}_{R} \not \partial \Psi_{R}\right)-\frac{1}{2}\left(\Psi_{L}^{T} C m_{L} \Psi_{L}+\Psi_{R}^{T} C m_{R} \Psi_{R}+\text { H.c. }\right) \\
& +i \bar{N}_{R} \not \partial N_{R}-\frac{1}{2}\left(N_{R}^{T} C m_{N} N_{R}+\text { H.c. }\right),
\end{aligned}
$$

where $m_{\Phi}^{2}>0$ in order to preserve $\left(\mathbb{Z}_{2}\right)_{\Phi} . m_{N}$ is a $n_{N} \times n_{N}$ matrix that, as in the case of the $\nu_{R}$, can be assumed to be diagonal; possible mechanisms for vector-boson-mass generation are reviewed in appendix B.

The last term in (4.2), responsible for dim-4 interactions between $\nu \mathrm{SM}$ and DM, reads

$$
\mathcal{L}_{\nu \mathrm{SM} \times \mathrm{DM}}^{(4)}=g_{\varphi} \varphi^{\dagger} \varphi \Phi^{2}+\left(\nu_{R}^{T} C Y_{\Phi} N_{R}+\text { H.c. }\right) \Phi,
$$

where $Y_{\Phi}$ is a $n_{\nu} \times n_{N}$ matrix.

Except of the standard Higgs portal, $\varphi^{\dagger} \varphi \Phi^{2}$, following [33, 34], we have added above a possible Yukawa interactions between $\nu_{R}, N_{R}$ and the dark scalar $\Phi$. Here we have also assumed that the dark fields can carry only their own $\mathbb{Z}_{2}$ quantum numbers, so a given dark field can not transform non-trivially under $\mathbb{Z}_{2}$ that stabilizes a different dark sector component, this eliminates some operators that otherwise would be present, e.g. $\nu_{R}^{T} C \Psi_{R} \Phi$. Note that the kinetic mixing between the $\mathrm{U}(1)_{Y}$ and the additional $\mathrm{U}(1)$ corresponding to the dark vector boson $V_{\mu}$ is forbidden by the stabilizing symmetry $\mathcal{G}_{\text {dark }}$. The Lagrangian $\mathcal{L}_{\nu \mathrm{SM} \times \mathrm{DM}}^{(4)}$ contains all possible renormalizable interactions between the $\nu \mathrm{SM}$ and $\mathrm{DM}$ that are allowed within the assumptions specified above.

We wish to make a comment concerning stability of fermions that appear in our scenario and which are neutral under SM gauge symmetries. We assumed that there are $n_{\nu}$ righthanded neutrinos $\nu_{R}$ that in general can decay by standard Yukawa interactions. Besides $\nu_{R}$ 's there are $n_{N}$ of $N_{R}$ 's, $\Psi_{R}$ and $\Psi_{L}$. Among then only $\Psi_{R}$ and $\Psi_{L}$ are guarantied to be stable (by the virtue of $\left(\mathbb{Z}_{2}\right)_{\Psi_{R}} \times\left(\mathbb{Z}_{2}\right)_{\Psi_{L}}$ ). Since both $N_{R}$ 's and $\Phi$ are odd under $\mathcal{G}_{\text {dark }}$, therefore the lightest of them is stable, in other words $N_{R}$ 's might be unstable.

Equations of motion for $\nu \mathrm{SM}$ fields derived from the $\mathcal{L}^{(4)}$ for $\nu \mathrm{SM}$ fields are the same as in the SM with two exceptions. Equations for the Higgs doublet $(\varphi)$ and right-handed neutrinos $\left(\nu_{R}\right)$ contain terms, that originate from interactions present in $\mathcal{L}_{\nu \mathrm{SM} \times \mathrm{DM}}^{(4)}$. The complete list of equations of motion for $\mathrm{DM}$ and $\mathrm{SM} \nu_{R}$ fields is:

$$
\begin{aligned}
\left(D_{\mu} D^{\mu} \varphi\right)^{j} & =m^{2} \varphi^{j}-\lambda\left(\varphi^{\dagger} \varphi\right) \varphi^{j}-\varepsilon_{j k} \bar{l}^{k} \Gamma_{\nu} \nu_{R}-\bar{e} \Gamma_{e}^{\dagger} l^{j}-\varepsilon_{j k} \bar{q}^{k} \Gamma_{u} u-\bar{d} \Gamma_{d}^{\dagger} q^{j}+g_{\varphi} \varphi \Phi^{2} \\
\left(D^{\rho} G_{\rho \mu}\right)^{A} & =g_{s}\left(\bar{q} \gamma_{\mu} T^{A} q+\bar{u} \gamma_{\mu} T^{A} u+\bar{d} \gamma_{\mu} T^{A} d\right) \\
\left(D^{\rho} W_{\rho \mu}\right)^{I} & =\frac{g}{2}\left(\varphi^{\dagger} i \overleftrightarrow{D}_{\mu}^{I} \varphi+\bar{l} \gamma_{\mu} \tau^{I} l+\bar{q} \gamma_{\mu} \tau^{I} q\right) \\
\partial^{\rho} B_{\rho \mu} & =g^{\prime} Y_{\varphi} \varphi^{\dagger} i \overleftrightarrow{D_{\mu}} \varphi+g^{\prime} \sum_{\psi \in\{l, e, q, u, d\}} Y_{\psi} \bar{\psi} \gamma_{\mu} \psi
\end{aligned}
$$

\footnotetext{
${ }^{2}$ Note that Dirac mass terms $\bar{\Psi}_{L} \psi_{R}$ and $\bar{\Psi}_{L} N_{R}$ are forbidden by $\mathcal{G}_{\text {dark }}$ symmetries.
} 


$$
\begin{aligned}
i \not D l & =\Gamma_{\nu} \nu_{R} \tilde{\varphi}+\Gamma_{e} e \varphi \\
i \not \partial \nu_{R} & =\Gamma_{\nu}^{\dagger} \tilde{\varphi}^{\dagger} l+m_{\nu} \nu_{R}^{C}-Y_{\Phi}^{\dagger} N_{R}^{C} \Phi, \\
i \not \partial N_{R} & =m_{N} N_{R}^{C}-Y_{\Phi}^{\dagger} \nu_{R}^{C} \Phi \\
i \not D e & =\Gamma_{e}^{\dagger} \varphi^{\dagger} l \\
i \not D q & =\Gamma_{u} u \tilde{\varphi}+\Gamma_{d} d \varphi \\
i \not D u & =\Gamma_{u}^{\dagger} \tilde{\varphi}^{\dagger} q \\
i \not D d & =\Gamma_{d}^{\dagger} \varphi^{\dagger} q, \\
\partial_{\mu} \partial^{\mu} \Phi & =-m_{\Phi} \Phi-\kappa \Phi^{3}+2 g_{\varphi} \Phi \varphi^{\dagger} \varphi+\nu_{R}^{T} C Y_{\Phi} N_{R}+\bar{N}_{R} C Y_{\Phi}^{\dagger} \bar{\nu}_{R}^{T}, \\
\partial^{\mu} V_{\mu \nu} & =-m_{V} V_{\nu}, \\
i \not \partial \Psi_{L, R} & =m_{L, R} \Psi_{L, R}^{c} .
\end{aligned}
$$

Many operators of the form $\nu \mathrm{SM} \times \mathrm{DM}$ are redundant through the application of the equations of motion [3-8], and should be omitted from the basis. Below we provide an illustration of this process of elimination; the notation we use is the following. If an operator includes l.h.s. of one of the above equations, then it can be written as a sum of the operators that consists of the r.h.s. of that equation and an operator, denoted by EOM, which vanishes due to that equation of motion. The purpose is to express a given operator as a linear combination of other operators, total derivatives TD and EOM. Such operators are redundant in effective Lagrangian. Operators vanishing due to the Bianchi identity are denoted by $\mathrm{BI}$. Using table 2 and 3 and these rules one can construct an irreducible basis of $\nu \mathrm{SM} \times \mathrm{DM}$ operators up to $\operatorname{dim} 6$.

We provide two examples of how the equations of motion can be used to eliminate some operators. First we show that $\bar{\psi} \gamma_{\mu} \psi \partial^{\mu}\left(\Phi^{2}\right)\left(\psi \in\left\{l, \nu_{R}, e, q, u, d\right\}\right)$ is redundant. After integrating by parts and applying equation of motion (4.7) we obtain the following $\bar{\psi} \gamma_{\mu} \psi \partial^{\mu}\left(\Phi^{2}\right)=\mathrm{TD}-\partial_{\mu}\left(\bar{\psi} \gamma_{\mu} \psi\right) \Phi^{2}=\mathrm{TD}-(\bar{\psi} \not D \psi+$ h.c. $) \Phi^{2}=\mathrm{TD}+\mathrm{EOM}+O_{\nu \mathrm{SM}}^{(4)} \times \Phi^{2}$, where $O_{\nu \mathrm{SM}}^{(4)} \times \Phi^{2}$ denotes operators made as a product of some operator belonging to $\mathcal{L}_{\nu \mathrm{SM}}^{(4)}$ and $\Phi^{2}$. If all operators $O_{\nu \mathrm{SM}}^{(4)} \times \Phi^{2}$ are included in our list then there is no need to have $\bar{\psi} \gamma_{\mu} \psi \partial^{\mu}\left(\Phi^{2}\right)$ as well.

A bit more involved algebra is needed to show redundancy of $B^{\mu \nu} \bar{\Psi} \gamma_{\mu} \partial_{\nu} \Psi$

$$
\begin{aligned}
B^{\mu \nu} \bar{\Psi} \gamma_{\mu} \partial_{\nu} \Psi= & \frac{1}{2} B^{\mu \nu} \bar{\Psi}\left(\gamma_{\mu} \gamma_{\nu} \not \partial+\gamma_{\mu} \not \partial \gamma_{\nu}\right) \Psi \\
= & \frac{1}{2} B^{\mu \nu} \bar{\Psi}\left(\gamma_{\mu} \gamma_{\nu} \not \partial-\not \partial \gamma_{\mu} \gamma_{\nu}\right) \Psi+B^{\mu \nu} \bar{\Psi} \gamma_{\nu} \partial_{\mu} \Psi \\
= & \frac{1}{4} B^{\mu \nu} \bar{\Psi}\left(\gamma_{\mu} \gamma_{\nu} \not \partial-\not \partial \gamma_{\mu} \gamma_{\nu}\right) \Psi \\
= & \frac{1}{4} B^{\mu \nu} \bar{\Psi} \gamma_{\mu} \gamma_{\nu} \not \partial \Psi+\frac{1}{4} \bar{\Psi} \overleftarrow{\not} \gamma_{\mu} \gamma_{\nu} \Psi B^{\mu \nu}+\frac{1}{4} \bar{\Psi} \gamma_{\rho} \gamma_{\mu} \gamma_{\nu} \Psi \partial^{\rho} B^{\mu \nu}+\mathrm{TD} \\
= & \mathrm{EOM}+\mathrm{TD}+\mathrm{BI} \\
& +\frac{1}{4}\left(B_{\mu \nu} \Psi^{T} C \sigma^{\mu \nu} \Psi+\text { H.c. }\right)+\frac{i g^{\prime}}{4} \varphi^{\dagger} \overleftrightarrow{D}{ }_{\mu} \varphi \bar{\Psi} \gamma^{\mu} \Psi+\frac{g^{\prime}}{2} \sum_{\psi \in\{l, e, q, u, d\}} Y_{\psi} \bar{\psi} \gamma_{\mu} \psi \bar{\Psi} \gamma^{\mu} \Psi
\end{aligned}
$$


where we used

$$
\begin{aligned}
\bar{\Psi} \gamma_{\rho} \gamma_{\mu} \gamma_{\nu} \Psi \partial^{\rho} B^{\mu \nu} & =2 \bar{\Psi} \gamma^{\nu} \Psi \partial^{\rho} B_{\rho \nu}-\bar{\Psi} i \varepsilon_{\rho \mu \nu \sigma} \gamma^{\sigma} \gamma_{5} \Psi \partial^{\rho} B^{\mu \nu} \\
& =\mathrm{EOM}+\mathrm{BI}+i g^{\prime} \varphi^{\dagger} \overleftrightarrow{D}_{\mu} \varphi \bar{\Psi} \gamma^{\mu} \Psi+2 g^{\prime} \sum_{\psi \in\{l, e, q, u, d\}} Y_{\psi} \bar{\psi} \gamma_{\mu} \psi \bar{\Psi} \gamma^{\mu} \Psi
\end{aligned}
$$

Again, if operators $i \varphi^{\dagger} \overleftrightarrow{D}_{\mu} \varphi \bar{\Psi} \gamma^{\mu} \Psi$ and $\bar{\psi}_{p} \gamma_{\mu} \psi_{q} \bar{\Psi} \gamma^{\mu} \Psi$ were present then $B^{\mu \nu} \bar{\Psi} \gamma_{\mu} \partial_{\nu} \Psi$ should be omitted from the operator basis. Similar arguments apply if $B^{\mu \nu}$ is replaced by $\tilde{B}^{\mu \nu}$.

Before proceeding to the final table collecting all the effective operators we discuss the mechanisms of dark vector boson mass generation, as they are relevant for the final output.

\subsection{The Stuckelberg mechanism}

In this scenario the Lagrangian (B.2) is invariant under $\mathbb{Z}_{2}$ symmetry, with both $\sigma$ and $V_{\mu}$ being odd. The equations of motion read

$$
\begin{aligned}
\partial_{\mu}\left(\partial^{\mu} \sigma-m_{V} V^{\mu}\right) & \equiv \partial_{\mu} \mathcal{V}^{\mu}=0 \\
\partial_{\mu} V^{\mu \nu} & =-m_{V}^{2} V^{\nu}+m_{V} \partial^{\nu} \sigma=m_{V} \mathcal{V}^{\nu}
\end{aligned}
$$

The Stuckelberg Lagrangian and equations of motion reduce to a part of the Lagrangian (4.4) and equations (4.8) when the $\sigma=0$ (unitary) gauge is adopted.

It is worth, at this point, to discuss in some detail the operator composed of two dark vector fields and two Higgs boson doublets: $V_{\mu} V^{\mu} \varphi^{\dagger} \varphi$, the effects of which have been investigated in the literature e.g. in [35] and [36]. Because of gauge invariance this operator can only be generated by $\mathcal{V}_{\mu} \mathcal{V}^{\mu} \varphi^{\dagger} \varphi$ of mass dimension 6 . It should be noticed that in the $\sigma=0$ gauge

$$
\frac{1}{\Lambda^{2}} \mathcal{V}_{\mu} \mathcal{V}^{\mu} \varphi^{\dagger} \varphi \rightarrow \frac{m_{V}^{2}}{\Lambda^{2}} V_{\mu} V^{\mu} \varphi^{\dagger} \varphi
$$

therefore there appears an unavoidable suppression factor $m_{V}^{2} / \Lambda^{2}$ even though formally $V_{\mu} V^{\mu} \varphi^{\dagger} \varphi$ is dim-4 operator. Note that higher dimensional operators of that sort would be suppressed by higher powers of $\Lambda$.

\subsection{The Higgs mechanism}

Another method to generate vector mass is the Higgs mechanism (B.3). In this case we start with a sector of the underlying theory containing a complex scalar field $\phi$ that we write in the form $\phi=(\rho / \sqrt{2}) \exp (i \sigma /\langle\rho\rangle)$ where $\langle\rho\rangle$ is the vacuum expectation value of $\rho$. In order to simplify arguments, we assume here that the Higgs-portal coupling $\lambda_{x} \phi^{\dagger} \phi \varphi^{\dagger} \varphi$ is weak, so $\lambda_{x} \ll 1$. Then, fluctuations of $\rho$ are mass-eigenstates that may belong to the heavy sector with their mass $\propto \Lambda$, so that they decouple from the low-energy theory, while $V_{\mu}$ and $\sigma$ might be light. The vector-boson mass $m_{V}=g\langle\rho\rangle$ is of order of the SM Higgs field vacuum expectation value $v$ provided the gauge coupling constant $g \sim v / \Lambda$ and $\langle\rho\rangle \sim \Lambda$. The stabilizing symmetry $\left(\mathbb{Z}_{2}\right)_{V}$ corresponds to charge conjugation under which $\sigma$ and $V_{\mu}$ are odd but $\rho$ is even; this ensures that the symmetry remains unbroken and $V_{\mu}$ is stable, for details see appendix B. 
Let us again focus on the $V_{\mu} V^{\mu} \varphi^{\dagger} \varphi$ operator, which is generated here within the underlying theory from the kinetic term for $\phi$ and the Higgs-portal coupling $\lambda_{x} \phi^{\dagger} \phi \varphi^{\dagger} \varphi$. Using the notation of appendix $\mathrm{B}$, we write $\rho=\langle\rho\rangle+\chi \simeq f+\chi$, where $\chi$ is a physical scalar of mass $m_{\chi}=\sqrt{2 \lambda_{\phi}} f+O\left(\lambda_{x}\right)$, and find that the Lagrangian contains the terms

$$
\frac{\chi}{f} m_{V}^{2} V_{\mu} V^{\mu}+\lambda_{x} \chi f \varphi^{\dagger} \varphi
$$

which upon integrating out the $\chi$ generate the operator

$$
\propto\left(\frac{m_{V}^{2}}{f} V_{\mu} V^{\mu}\right) \frac{1}{m_{\chi}^{2}}\left(\lambda_{x} f \varphi^{\dagger} \varphi\right) \simeq \lambda_{x}\left(\frac{m_{V}^{2}}{m_{\chi}^{2}}\right) V_{\mu} V^{\mu} \varphi^{\dagger} \varphi
$$

Note that the resulting operator received similar suppression as in the case of the Stuckelberg approach (4.13). It should be emphasized that the suppression factor is always proportional to $\lambda_{x}\left(m_{V}^{2} / m_{\chi}^{2}\right)$, even if the Higgs-portal coupling is not weak, as it was assumed above just for simplicity.

Few other comments are here in order. First, note that the operator $\phi^{*} D_{\mu} D^{\mu} \phi$ is not invariant under $\left(\mathbb{Z}_{2}\right)_{V}$, unless we add its conjugate:

$$
\left(\phi^{*} D_{\mu} D^{\mu} \phi+\left(D_{\mu} D^{\mu} \phi\right)^{*} \phi\right) \varphi^{\dagger} \varphi .
$$

Similarly $\left(D_{\mu} \phi\right)^{*} D_{\nu} \phi+\left(D_{\nu} \phi\right)^{*} D_{\mu} \phi$ is invariant under $\left(\mathbb{Z}_{2}\right)_{V}$. Note that this operator is symmetric in its Lorentz indices and vanishes after contraction with $B_{\mu \nu}$.

\section{$4.3 \quad \nu \mathrm{SM} \times \mathrm{DM}$ operators}

The resulting effective operators obtained via (1.1) using tables 2 and 3 are contained in table 4 . There are several comments here in order.

The effective operators were divided in table 4 into operators that can be generated at the tree level ("TREE") and those requiring loops ("LOOP"). For this purpose we extended the method used to categorize the Standard Model effective operators [41] to the case of operators including dark matter fields [29]. Note that operators denoted by "TREE" are those for which there exists a new physics model where they are generated at the tree level, but it is not yet possible to determine whether this is the case for the situation realized in Nature; a specific model may generate those potentially tree generated (PTG) operators at one or higher loops, or may not generate it at all because of the details of its particle content and symmetries.

The operators denoted by ("LOOP") cannot be generated by tree graphs for the following reasons. First note that tree graphs with three external light fields and an exchange of heavy mediator do not exist, therefor operators composed of just three fields require loops. In the case of operators built of vector field tensors one cannot find relevant renormalizable vertices that would join a mediator with two field strength tensors or with one field strenght tensor and additional field. Therefore operators containing field tensors also require loops. Finally, in order to generate $V_{\mu} V^{\mu} \varphi^{\dagger} \varphi$ operator at the tree level within the Stuckelberg scenario one needs a presence of a mediator which would be charged under the Stuckelberg gauge symmetry and in addition which would have a non-zero vev. That 


\begin{tabular}{|c|c|c|c|c|c|}
\hline & 1 & $\Lambda^{-1}$ & \multicolumn{3}{|c|}{$\Lambda^{-2}$} \\
\hline & & & $\Phi, N_{R}$ & $\Psi$ & $V_{\mu}$ \\
\hline Tree: & $\begin{array}{c}\varphi^{\dagger} \varphi \Phi^{2} \\
\nu_{R}^{T} C N_{R} \Phi\end{array}$ & $\begin{array}{c}\varphi^{\dagger} \varphi \Psi^{T} C \Psi \\
\bar{l} \tilde{\varphi} N_{R} \Phi \\
\nu_{R}^{T} C \nu_{R} \Phi^{2}\end{array}$ & $\begin{array}{c}\varphi^{\dagger} \varphi \partial_{\mu} \Phi \partial^{\mu} \Phi \\
\varphi^{\dagger} \varphi \Phi^{4} \\
\left(\varphi^{\dagger} \varphi\right)^{2} \Phi^{2} \\
\bar{l} \nu_{R} \tilde{\varphi} \Phi^{2} \\
\bar{l} e \varphi \Phi^{2} \\
\bar{q} u \tilde{\varphi} \Phi^{2} \\
\bar{q} d \varphi \Phi^{2} \\
\nu_{R}^{T} C N_{R} \Phi^{3} \\
\varphi^{\dagger} \varphi \nu_{R}^{T} C N_{R} \Phi \\
N_{R}^{T} C \gamma^{\mu} l \varepsilon \partial_{\mu} \varphi \Phi\end{array}$ & $\begin{array}{c}\nu_{R}^{T} C \nu_{R} \Psi^{T} C \Psi \\
\nu_{R}^{T} C \sigma^{\mu \nu} \nu_{R} \Psi^{T} C \sigma_{\mu \nu} \Psi \\
\nu_{R}^{T} C \nu_{R} \bar{\Psi} C \bar{\Psi}^{T} \\
\nu_{R}^{T} C \sigma^{\mu \nu} \nu_{R} \bar{\Psi} \sigma_{\mu \nu} C \bar{\Psi}^{T} \\
\bar{\psi}_{p} \gamma_{\mu} \psi_{q} \bar{\Psi} \gamma^{\mu} \Psi \\
i \varphi^{\dagger} \overleftrightarrow{D}_{\mu} \varphi \bar{\Psi} \gamma^{\mu} \Psi\end{array}$ & $m_{V}^{2} \varphi^{\dagger} \varphi V_{\mu} V^{\mu}$ \\
\hline LOOP: & & $\stackrel{(\sim)}{B}_{\mu \nu} \Psi^{T} C \sigma^{\mu \nu} \Psi$ & $\begin{array}{c}\nu_{R}^{T} C \partial_{\mu} N_{R} \partial^{\mu} \Phi \\
\nu_{R}^{T} C \sigma^{\mu \nu} N_{R} \stackrel{(\sim)}{B}_{\mu \nu} \Phi \\
\quad \stackrel{(\sim)}{X}_{\mu \nu} X^{\mu \nu} \Phi^{2}\end{array}$ & & $\begin{array}{l}\varphi^{\dagger} \varphi \stackrel{(\sim)}{V}_{\mu \nu} V^{\mu \nu} \\
m_{V}^{2} \varphi^{\dagger} \varphi V_{\mu} V^{\mu}\end{array}$ \\
\hline
\end{tabular}

Table 4. List of all $\nu \mathrm{SM} \times \mathrm{DM}$ operators up to $\operatorname{dim} 6$, that are suppressed by at most $\Lambda^{-2}$. Dark matter sector consists of a real scalar $\Phi$, chiral fermions $\Psi \in\left\{\Psi_{L}, \Psi_{R}, N_{R}\right\}$ and vector field $V_{\mu}$. Tree and loop-generated operators are collected in the upper and lower part of the table, respectively. Operator $\varphi^{\dagger} \varphi V_{\mu} V^{\mu}$ appears in both categories, because within the Higgs mechanism, it can be generated at the tree-level approximation, while within the Stuckelberg model it requires a loop. Note that one entry in the table may refer to various operators, because $(\sim)$ over $X_{\mu \nu}$ denotes $X_{\mu \nu}$ or $\tilde{X}_{\mu \nu}, X_{\mu \nu}$ stands for $B_{\mu \nu}, W_{\mu \nu}^{I}$ or $G_{\mu \nu}^{A}$ and $\psi \in\left\{l, \nu_{R}, e, q, u, d\right\}$. The bosonic operators are all Hermitian. In case of the operators containing fermions, $i \varphi^{\dagger} \overleftrightarrow{D}_{\mu} \varphi \bar{\Psi} \gamma^{\mu} \Psi$ is Hermitian and conjugation of $\bar{\psi}_{p} \gamma_{\mu} \psi_{q} \bar{\Psi} \gamma^{\mu} \Psi$ is equivalent to transposition of the generation indices. For the remaining operators Hermitian conjugations are not listed explicitly.

however would mean a contribution to the dark vector mass from the vev, so it would be the Higgs mechanism not the Stuckelberg one. Therefore within the Stuckelberg scenario there is no way to generate the $V_{\mu} V^{\mu} \varphi^{\dagger} \varphi$ operator at the tree level.

All operators present in table 4 are of the form of (1.1) with $\mathcal{O}_{\nu \mathrm{SM}}$ and $\mathcal{O}_{\mathrm{DM}}$ being separately invariant under symmetries of $\nu \mathrm{SM}$ and $\mathcal{G}_{\text {dark }}$, respectively. Such operators can be generated at tree-level by the exchange of heavy particles that may or may not be neutral under the $\nu \mathrm{SM}$ and dark symmetries (both options always exist, though existing data may constrain the properties of non-neutral particles more severely). For example $\varphi^{\dagger} \varphi \Psi_{L}^{T} C \Psi_{L}$ can be generated by the exchange of a neutral heavy scalar $S$ with couplings $S|\varphi|^{2}$ and $\Psi_{L}^{T} C \Psi_{L} S$; or by a heavy Dirac fermion $F$ with the same SM gauge transformation properties as $\varphi$, odd under $\left(\mathbb{Z}_{2}\right)_{\Psi_{L}}$, and with couplings $\bar{F} \varphi \Psi_{L}$ and $\bar{F} \varphi \Psi_{L}^{C}$ (C denotes the usual charge conjugation operation). For an illustration, in table 5 , we draw generic diagrams (within an underlying theory) that could be responsible for operators contained in table 4 .

It should be noticed that the stabilizing symmetries imply that neither $\Psi_{L}$ nor $\Psi_{R}$ can appear separately in any operators. Allowed interactions beetwen the Standard Model 


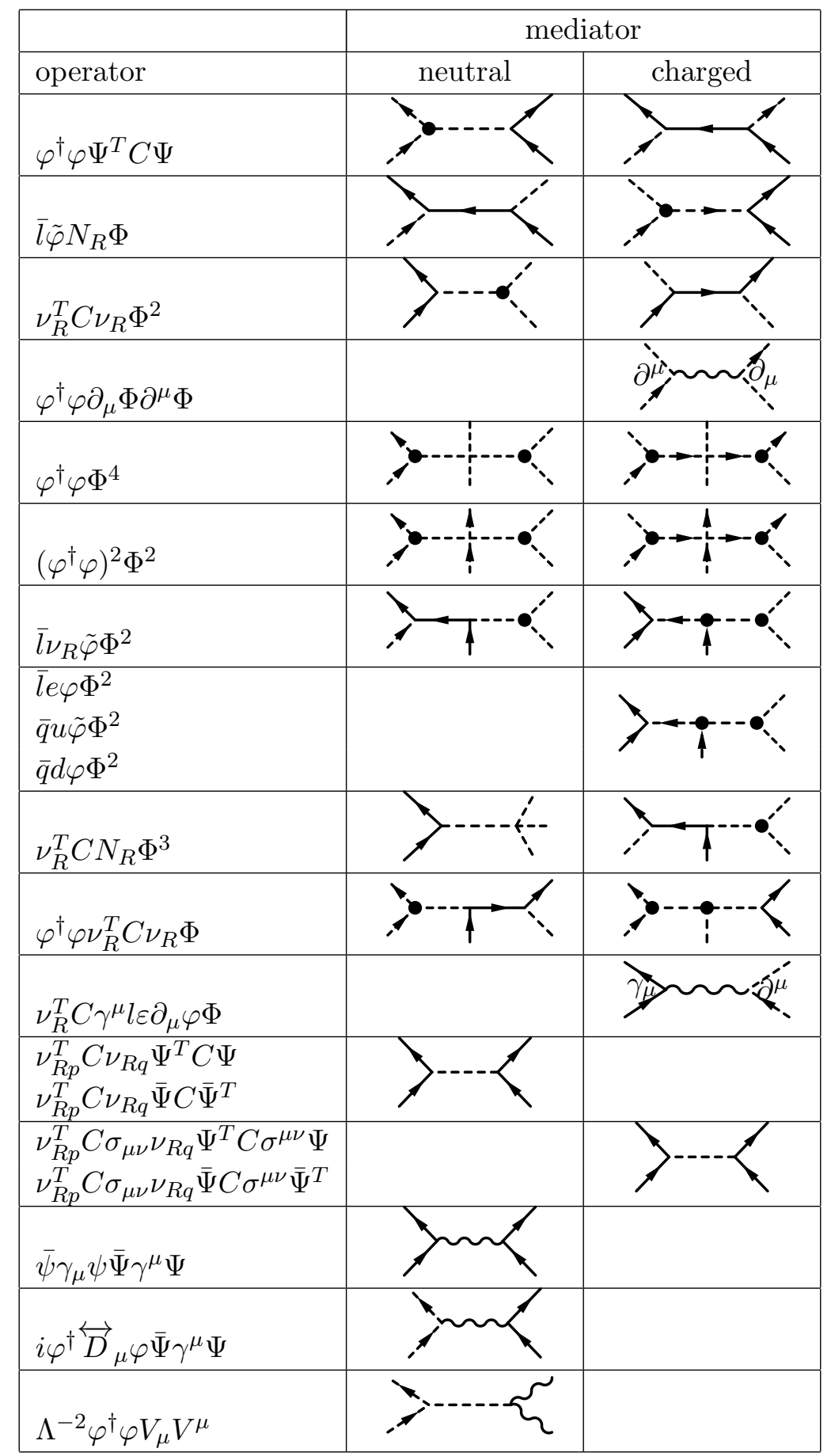

Table 5. The table shows illustrative diagrams that source tree-level generated (PTG) operators contained in table 4 . For most cases we present both diagrams generated by an exchange of a mediator that is neutral (second column) and/or charged (third column) under dark and $\nu \mathrm{SM}$ symmetries. A thick dot stands for a dimensionful cubic scalar coupling of the order of $\Lambda$, external lines correspond to $\nu \mathrm{SM}$ fields while internal ones describe propagators of heavy mediators. Dashed, dashed with arrows, solid and wavy lines correspond to real scalars, complex scalars, fermions and vector bosons, respectively. 
and pairs of dark fermions are the same in the case of left and right-chiral fields, therefore one can use just $\Psi$ to denote all of them. The operators with $\Psi_{L}$ and $\Psi_{R}$ form disjoint sets, consequently one could drop e.g. $\Psi_{R}$, such that $\Psi$ in table 4 corresponds both to $\Psi_{L}$ and $N_{R}$.

\section{Summary}

In this paper we have constructed a basis of operators of dim $\leq 6$ which describe interactions between Dark Matter composed of an Abelian vector, chiral fermions and a real scalar with the Standard Model. Our assumptions were the following:

- Each component of the dark sector is stable by the virtue of an independent $\mathbb{Z}_{2}$ symmetry,

- Each component of the dark sector transforms non-trivially only under the symmetry which is responsible for its own stability,

- The Standard Model fields are neutral under any symmetries of the dark sector,

- The dark sector contains neutral fermions, $N_{R}$, which are odd under $\left(\mathbb{Z}_{2}\right)_{\Phi}$ symmetry responsible for stability of $\Phi$, the $N_{R}$ has no other quantum numbers,

- The dark sector fields are neutral under any symmetry of the Standard Model.

The basis consistent with the above assumptions is presented in table 4 . where operators redundant under the application of the equations of motion have been eliminated.

We have shown that there exist only two possible operators of dim- 4 that are consistent with our assumptions: the Higgs portal $\varphi^{\dagger} \varphi \Phi^{2}$ and Yukawa interactions, $\nu_{R}^{T} C N_{R} \Phi$.

Note added. After completing this paper we have learned about the papers [39, 40], where the operator basis for interactions between the Standard Model and dark matter had been also constructed. Assumptions made here and in $[39,40]$ were different. The operators in [39, 40] agree with ours whenever the assumptions and field content in both papers coincide.

\section{Acknowledgments}

BG is partially supported by the National Science Centre (Poland) under research project, decision no DEC-2011/01/B/ST2/00438.

\section{A Conventions and definitions}

In this appendix we collect useful formulae and specify conventions adopted in the main text. $\tilde{\varphi}$ is defined as $\tilde{\varphi}_{i} \equiv \varepsilon_{i j}\left(\varphi^{j}\right)^{*}$. Tensors $\varepsilon_{i j}$ and $\varepsilon_{\mu \nu \rho \sigma}$ are totally antisymmetric with $\varepsilon_{12}=+1, \varepsilon_{0123}=+1$. Dual tensor to $X_{\mu \nu}$ is defined as $\tilde{X}_{\mu \nu}=\frac{1}{2} \varepsilon_{\mu \nu \rho \sigma} X^{\rho \sigma}$. Symbol $(\sim)$ over $X$ denotes $X$ or $\tilde{X}$. Metric signature $(+,-,-,-)$ is chosen. 
Sign convention for covariant derivative is exemplified by

$$
\left(D_{\mu} q\right)^{\alpha j}=\left[\left(\partial_{\mu}+i g^{\prime} Y_{q} B_{\mu}\right) \delta^{\alpha \beta} \delta^{j k}+i g_{s} T^{A \alpha \beta} G_{\mu}^{A} \delta^{j k}+i g S^{I j k} W_{\mu}^{I} \delta^{\alpha \beta}\right] q^{\beta k},
$$

where $T^{A}=\frac{1}{2} \lambda^{A}$ are $\mathrm{SU}(3)$ generators with Gell-Mann matrices $\lambda^{A}$ and $S^{I}=\frac{1}{2} \tau^{I}$ are $\mathrm{SU}(2)$ generators with Pauli matrices $\tau^{I}$. It is useful to define Hermitian derivative term

$$
i \varphi^{\dagger} \overleftrightarrow{D}_{\mu} \varphi \equiv i \varphi^{\dagger} D_{\mu} \varphi-i\left(D_{\mu} \varphi\right)^{\dagger} \varphi
$$

Gauge field strength tensors and their covariant derivatives are

$$
\begin{aligned}
G_{\mu \nu}^{A} & =\partial_{\mu} G_{\nu}^{A}-\partial_{\nu} G_{\mu}^{A}-g_{s} f^{A B C} G_{\mu}^{B} G_{\nu}^{C}, & & \left(D_{\rho} G_{\mu \nu}\right)^{A}=\partial_{\rho} G_{\mu \nu}^{A}-g_{s} f^{A B C} G_{\rho}^{B} G_{\mu \nu}^{C}, \\
W_{\mu \nu}^{I} & =\partial_{\mu} W_{\nu}^{I}-\partial_{\nu} W_{\mu}^{i}-g \varepsilon^{I J K} W_{\mu}^{J} W_{\nu}^{K}, & & \left(D_{\rho} W_{\mu \nu}\right)^{I}=\partial_{\rho} W_{\mu \nu}^{I}-g \varepsilon^{I J K} W_{\rho}^{J} W_{\mu \nu}^{K}, \\
B_{\mu \nu} & =\partial_{\mu} B_{\nu}-\partial_{\nu} B_{\mu}, & D_{\rho} B_{\mu \nu} & =\partial_{\rho} B_{\mu \nu} .
\end{aligned}
$$

\section{B Mass generation for Abelian vector bosons}

In this appendix we review possible mechanisms of Abelian vector-boson mass generation. A massive vector field can be described by the Proca Lagrangian (B.1), since the mass term spoils the gauge invariance therefore renormalizabilty of this theory is not apparent.

$$
\mathcal{L}_{P}=-\frac{1}{4} V_{\mu \nu} V^{\mu \nu}+\frac{1}{2} m_{V}^{2} V_{\mu} V^{\mu} \quad \text { for } \quad V_{\mu \nu}=\partial_{\mu} V_{\nu}-\partial_{\nu} V_{\mu}
$$

\section{B.1 The Stuckelberg mechanism}

The Stuckelberg mechanism is a way to restore the gauge symmetry of (B.1) by introducing a real scalar field $\sigma$ (see e.g. [37, 38]) with appropriate transformation rules:

$$
\begin{aligned}
\mathcal{L}_{S} & =-\frac{1}{4} V_{\mu \nu} V^{\mu \nu}+\frac{1}{2}\left(\partial_{\mu} \sigma-m_{V} V_{\mu}\right)\left(\partial^{\mu} \sigma-m_{V} V^{\mu}\right), \\
V_{\mu} \rightarrow V_{\mu}^{\prime} & =V_{\mu}+\partial_{\mu} \alpha, \\
\sigma \rightarrow \sigma^{\prime} & =\sigma+m_{V} \alpha .
\end{aligned}
$$

The field $\sigma$ can be eliminated from the model by choosing $\alpha=-\sigma / m_{V}$; in this gauge the Stuckelberg Lagrangian becomes the same as in the Proca theory (B.1). It should be emphasized that when the mass of the vector field is generated by the Stuckelberg mechanism (B.2) the gauge invariance requires that the vector field appears only as $V_{\mu \nu}$, $\tilde{V}_{\mu \nu}$ or $\mathcal{V}_{\mu}=\partial_{\mu} \sigma-m_{V} V_{\mu}$, both of which have mass dimension 2 .

\section{B.2 The Higgs mechanism}

Another way to make an Abelian vector field massive is the Higgs mechanism. It uses complex scalar field $\phi$ that acquires a vacuum expectation value $\langle\phi\rangle$ which spontaneously breaks the $\mathrm{U}(1)$ local symmetry and thus generates a mass $m_{V}=g f$ for the associated gauge vector field $V_{\mu}$ :

$$
\begin{aligned}
& \mathcal{L}_{\phi}=-\frac{1}{4} V_{\mu \nu} V^{\mu \nu}+\left(D_{\mu} \phi\right)^{\dagger} D^{\mu} \phi-\lambda_{\phi}\left(\phi^{\dagger} \phi-\frac{f^{2}}{2}\right)^{2} \text { for } D_{\mu} \phi=\left(\partial_{\mu}-i g V_{\mu}\right) \phi \\
& V_{\mu} \rightarrow V_{\mu}^{\prime}-\partial_{\mu} \alpha, \quad \phi \rightarrow e^{i g \alpha} \phi^{\prime} .
\end{aligned}
$$


In contrast to the Stuckelberg mechanism the scalar field cannot be completely eliminated by the gauge transformation.

Writing the complex scalar field in the form $\phi=(\rho / \sqrt{2}) \exp (i \sigma / f)$ the Lagrangian becomes

$$
\mathcal{L}_{\phi}=-\frac{1}{4} V_{\mu \nu} V^{\mu \nu}+\frac{1}{2} \partial_{\mu} \rho \partial^{\mu} \rho+\frac{1}{2}\left(\frac{\rho}{f}\right)^{2}\left(\partial_{\mu} \sigma-m_{V} V_{\mu}\right)\left(\partial^{\mu} \sigma-m_{V} V^{\mu}\right)-\frac{1}{4} \lambda_{\phi}\left(\rho^{2}-f^{2}\right)^{2} .
$$

$\rho$ then acquires a vacuum expectation value, $\langle\rho\rangle=f$ and $\sigma$ plays the same role as the corresponding field in the Stuckelberg approach. Writing $\rho=f+\chi$ we find that $\chi$ has a mass $m_{\chi}=\sqrt{2 \lambda_{\phi}} f$ while $\sigma$ is massless (though, of course, one can always choose a gauge where $\sigma$ vanishes). In the limit $f \rightarrow \infty, g \rightarrow 0$ with $m_{V}$ kept fixed the $\chi$ decouples and we recover the Stuckelberg Lagrangian (B.2).

As mentioned previously we will assume that $\chi$ is in the heavy sector (so that its mass $m_{\chi} \sim \Lambda$ ) while $V_{\mu}$ should remain in the dark sector. We implement this scenario by taking $\lambda_{\phi}=O(1), f=O(\Lambda)$ and $g \ll 1$, for example $g \sim v / \Lambda$ for a $V_{\mu}$ with a mass of the order of the electroweak scale.

In (B.3) we have omitted the Higgs-portal term $\lambda_{x} \phi^{\dagger} \phi \varphi^{\dagger} \varphi$, which affects the VEVs and introduces a scalar mixing. However, if the previous assumptions are kept, in particular when $f=\langle\phi\rangle=O(\Lambda)$, we still obtain the scenario with $V_{\mu}$ in the light sector and heavy $\chi$, which is then the mass eigenstate of $m_{\chi}=\sqrt{2 \lambda_{\phi}} f+O\left(\lambda_{x}\right)$.

Within the Higgs approach gauge invariant quantities containing a vector field are built from $V_{\mu \nu}, \tilde{V}_{\mu \nu}$ and covariant derivatives of complex scalar field $D_{\mu} \phi$. It is assumed that $\nu \mathrm{SM}$ fields are singlets under the Higgs U(1) symmetry. Operators built of $V_{\mu \nu}$ and $\tilde{V}_{\mu \nu}$ only are the same as in Stuckelberg case. Operators with $\phi$ appear at dimension 3 (or higher), because they must contain $\phi^{*}$ to ensure gauge invariance and at least one covariant derivative that contains the vector field.

Open Access. This article is distributed under the terms of the Creative Commons Attribution License (CC-BY 4.0), which permits any use, distribution and reproduction in any medium, provided the original author(s) and source are credited.

\section{References}

[1] S. Bhattacharya, A. Drozd, B. Grzadkowski and J. Wudka, Two-component dark matter, JHEP 10 (2013) 158 [arXiv:1309.2986] [INSPIRE].

[2] S. Bhattacharya, A. Drozd, B. Grzadkowski and J. Wudka, Constraints on two-component dark matter, Acta Phys. Polon. B 44 (2013) 2373 [arXiv:1310.7901] [inSPIRE].

[3] H.D. Politzer, Power corrections at short distances, Nucl. Phys. B 172 (1980) 349 [INSPIRE].

[4] H. Kluberg-Stern and J.B. Zuber, Renormalization of non-Abelian gauge theories in a background-field gauge. II. Gauge-invariant operators, Phys. Rev. D 12 (1975) 3159 [INSPIRE].

[5] C. Grosse-Knetter, Effective Lagrangians with higher derivatives and equations of motion, Phys. Rev. D 49 (1994) 6709 [hep-ph/9306321] [INSPIRE]. 
[6] C. Arzt, Reduced effective Lagrangians, Phys. Lett. B 342 (1995) 189 [hep-ph/9304230] [INSPIRE].

[7] H. Simma, Equations of motion for effective Lagrangians and Penguins in rare B decays, Z. Phys. C 61 (1994) 67 [hep-ph/9307274] [INSPIRE].

[8] J. Wudka, Electroweak effective Lagrangians, Int. J. Mod. Phys. A 9 (1994) 2301 [hep-ph/9406205] [INSPIRE].

[9] J. Hisano, D. Kobayashi, N. Mori and E. Senaha, Effective interaction of electroweak-interacting dark matter with Higgs boson and its phenomenology, Phys. Lett. B 742 (2015) 80 [arXiv:1410.3569] [INSPIRE].

[10] R.J. Hill and M.P. Solon, Standard model anatomy of WIMP dark matter direct detection. II. QCD analysis and hadronic matrix elements, Phys. Rev. D 91 (2015) 043505 [arXiv: 1409.8290] [INSPIRE].

[11] S. Matsumoto, S. Mukhopadhyay and Y.-L.S. Tsai, Singlet Majorana fermion dark matter: a comprehensive analysis in effective field theory, JHEP 10 (2014) 155 [arXiv:1407.1859] [INSPIRE].

[12] M.B. Krauss, S. Morisi, W. Porod and W. Winter, Higher dimensional effective operators for direct dark matter detection, JHEP 02 (2014) 056 [arXiv: 1312.0009] [INSPIRE].

[13] N.F. Bell, Y. Cai and A.D. Medina, Co-annihilating dark matter: effective operator analysis and collider phenomenology, Phys. Rev. D 89 (2014) 115001 [arXiv:1311.6169] [INSPIRE].

[14] E. Dudas, L. Heurtier, Y. Mambrini and B. Zaldivar, Extra U(1), effective operators, anomalies and dark matter, JHEP 11 (2013) 083 [arXiv:1307.0005] [INSPIRE].

[15] M.R. Buckley, Using effective operators to understand CoGeNT and CDMS-Si signals, Phys. Rev. D 88 (2013) 055028 [arXiv: 1308.4146] [INSPIRE].

[16] A. De Simone, A. Monin, A. Thamm and A. Urbano, On the effective operators for dark matter annihilations, JCAP 02 (2013) 039 [arXiv:1301.1486] [INSPIRE].

[17] J.-Y. Chen, E.W. Kolb and L.-T. Wang, Dark matter coupling to electroweak gauge and Higgs bosons: an effective field theory approach, Phys. Dark Univ. 2 (2013) 200 [arXiv: 1305.0021] [INSPIRE].

[18] R. Ding, Y. Liao, J.-Y. Liu and K. Wang, Comprehensive constraints on a spin-3/2 singlet particle as a dark matter candidate, JCAP 05 (2013) 028 [arXiv: 1302.4034] [INSPIRE].

[19] A. Greljo, J. Julio, J.F. Kamenik, C. Smith and J. Zupan, Constraining Higgs mediated dark matter interactions, JHEP 11 (2013) 190 [arXiv:1309.3561] [INSPIRE].

[20] Y.J. Chae and M. Perelstein, Dark matter search at a linear collider: effective operator approach, JHEP 05 (2013) 138 [arXiv:1211.4008] [INSPIRE].

[21] J.-M. Zheng et al., Constraining the interaction strength between dark matter and visible matter: I. Fermionic dark matter, Nucl. Phys. B 854 (2012) 350 [arXiv:1012.2022] [INSPIRE].

[22] Z.-H. Yu et al., Constraining the interaction strength between dark matter and visible matter: II. Scalar, vector and spin-3/2 dark matter, Nucl. Phys. B 860 (2012) 115 [arXiv: 1112.6052] [INSPIRE].

[23] R. Ding and Y. Liao, Spin 3/2 particle as a dark matter candidate: an effective field theory approach, JHEP 04 (2012) 054 [arXiv:1201.0506] [INSPIRE]. 
[24] K. Cheung, P.-Y. Tseng, Y.-L.S. Tsai and T.-C. Yuan, Global constraints on effective dark matter interactions: relic density, direct detection, indirect detection and collider, JCAP 05 (2012) 001 [arXiv: 1201.3402] [INSPIRE].

[25] Q.-H. Cao, C.-R. Chen, C.S. Li and H. Zhang, Effective dark matter model: relic density, CDMS II, Fermi LAT and LHC, JHEP 08 (2011) 018 [arXiv:0912.4511] [InSPIRE].

[26] J. Goodman et al., Constraints on light Majorana dark matter from colliders, Phys. Lett. B 695 (2011) 185 [arXiv: 1005.1286] [INSPIRE].

[27] M. Beltrán, D. Hooper, E.W. Kolb, Z.A.C. Krusberg and T.M.P. Tait, Maverick dark matter at colliders, JHEP 09 (2010) 037 [arXiv: 1002 .4137] [INSPIRE].

[28] J. Goodman et al., Constraints on dark matter from colliders, Phys. Rev. D 82 (2010) 116010 [arXiv: 1008.1783] [InSPIRE].

[29] M. Duch, Effective operators for dark matter interactions, MSc Thesis, University of Warsaw, Warsaw Poland (2014) [arXiv:1410.4427] [INSPIRE].

[30] W. Buchmüller and D. Wyler, Effective Lagrangian analysis of new interactions and flavor conservation, Nucl. Phys. B 268 (1986) 621 [INSPIRE].

[31] B. Grzadkowski, M. Iskrzynski, M. Misiak and J. Rosiek, Dimension-six terms in the standard model Lagrangian, JHEP 10 (2010) 085 [arXiv: 1008.4884] [INSPIRE].

[32] M.B. Einhorn and J. Wudka, The bases of effective field theories, Nucl. Phys. B 876 (2013) 556 [arXiv: 1307.0478] [inSPIRE].

[33] B. Grzadkowski and J. Wudka, Pragmatic approach to the little hierarchy problem: the case for dark matter and neutrino physics, Phys. Rev. Lett. 103 (2009) 091802 [arXiv:0902.0628] [INSPIRE].

[34] B. Grzadkowski and J. Wudka, Naive solution of the little hierarchy problem and its physical consequences, Acta Phys. Polon. B 40 (2009) 3007 [arXiv:0910.4829] [InSPIRE].

[35] O. Lebedev, H.M. Lee and Y. Mambrini, Vector Higgs-portal dark matter and the invisible Higgs, Phys. Lett. B 707 (2012) 570 [arXiv:1111.4482] [INSPIRE].

[36] S. Kanemura, S. Matsumoto, T. Nabeshima and N. Okada, Can WIMP dark matter overcome the nightmare scenario?, Phys. Rev. D 82 (2010) 055026 [arXiv:1005.5651] [INSPIRE].

[37] H. Ruegg and M. Ruiz-Altaba, The Stueckelberg field, Int. J. Mod. Phys. A 19 (2004) 3265 [hep-th/0304245] [INSPIRE].

[38] H. van Hees, The renormalizability for massive Abelian gauge field theories revisited, hep-th/0305076 [INSPIRE].

[39] J.F. Kamenik and C. Smith, FCNC portals to the dark sector, JHEP 03 (2012) 090 [arXiv: 1111.6402] [INSPIRE].

[40] J.F. Kamenik and C. Smith, Could a light Higgs boson illuminate the dark sector?, Phys. Rev. D 85 (2012) 093017 [arXiv: 1201.4814] [INSPIRE].

[41] C. Arzt, M.B. Einhorn and J. Wudka, Patterns of deviation from the standard model, Nucl. Phys. B 433 (1995) 41 [hep-ph/9405214] [InSPIRE]. 